\title{
Analysis and Visualization of Nerve Vessel Contacts for Neurovascular Decompression
}

\author{
Jochen Süßmuth ${ }^{1}$, Alexander Piazza ${ }^{1}$, Frank Enders ${ }^{1,2}$, Ramin Naraghi ${ }^{2}$, \\ Günther Greiner ${ }^{1}$, Peter Hastreiter ${ }^{1,2}$ \\ ${ }^{1}$ Computer Graphics Group, University of Erlangen-Nuremberg, Germany \\ ${ }^{2}$ Dept. of Neurosurgery, Neurocenter, University of Erlangen-Nuremberg, Germany \\ suessmuth@informatik.uni-erlangen.de
}

\begin{abstract}
Neurovascular compression syndromes are caused by a pathological contact between cranial nerves and vascular structures at the surface of the brainstem. Aiming at improved pre-operative analysis of the target structures, we propose calculating distance fields to provide quantitative information of the important nerve-vessel contacts. Furthermore, we suggest reconstructing polygonal models for the nerves and vessels. Color-coding with the respective distance information is used for enhanced visualization. Overall, our new strategy contributes to a significantly improved clinical understanding.
\end{abstract}

\section{Introduction}

The detailed understanding of the complex anatomical relationships between nerves and vessels at the surface of the brainstem is of major importance for the understanding of neurovascular compression (NVC) syndromes [1]. Associated with diseases such as trigeminal neuralgia (TN) and hemifacial spasm (HS), they are characterized by a pathological contact between vessels and the root entry or exit zone of cranial nerves. Its correct assessment is crucial for diagnosis and therapy planning.

Visualization of strongly T2 weighted MR (magnetic resonance) images based on the CISS (constructive interference in steady state) sequence has been shown to be a powerful tool for the analysis of NVC syndromes $[2,3]$. In order to overcome problems related to imaging artifacts, previous work suggested to consider vascular information from MR angiography (MRA) as additional source of information [4]. Based on the fusion of MRA and MR-CISS, advanced visualization was achieved [5]. The rendering quality was further improved with volume splatting and 2D transfer functions [6]. In a more recent contribution, polygonal reconstruction of neurovascular relationships and differentiation of arteries and veins based on additional MR imaging was suggested [7]. However, a software based detailed analysis of the nerve-vessel relationships is missing so far.

Aiming at an improved analysis of the nerve-vessel contacts in NVC syndromes, we suggest the computation of distance fields which store the spacing to the closest vascular structure. As input data, we use a combination of registered 
MR-CISS and MRA-TOF (time of flight) volumes. For enhanced visualization, we reconstruct polygonal models of the target structures, which are color-coded with the information retrieved from the distance field. As a general achievement, our approach results in a considerably improved qualitative analysis and allows for a precise detection and evaluation of the nerve-vessel contacts in NVC syndomes.

\section{Materials and methods}

\subsection{Data acquisition and preprocessing}

All data sets consisted of $512 \times 512 \times 144$ voxels with a size of $0.39 \times 0.39 \times$ $0.4 \mathrm{~mm}^{3}$. As illustrated in figure 1, MR-CISS data shows a high degree of contrast between hyperintense CSF (cerebrospinal fluid) and hypointense cranial nerves. On the other hand, the representation of the larger vessels is ambiguous due to flow artifacts. This drawback is circumvented by additionally considering vascular information obtained from MRA-TOF. In a preprocessing step, the volumes are rigidly registered using an implementation based on normalized mutual information [5]. Then, we use a semi-automatic segmentation to extract subvolumes of the cranial nerves and the brainstem from the MR-CISS data [2] (Fig. 1(right)).

\subsection{Polygonal reconstruction of nerves and vessels}

For improved analysis and subsequent visualization, we compute polygonal models of the vascular structures, the brainstem and the cranial nerves. This is achieved by extracting iso-surfaces for the vessels from the MRA-TOF data and for the nerves and the brainstem from the segmented MR-CISS data using marching cubes.

To reduce the typical aliasing artifacts introduced by the marching cubes algorithm, we apply a series of smoothing steps to the extracted meshes of the nerves and vessels. Our experiments showed that the second order umbrellaoperator $U^{2}(\mathbf{p})$ introduced by Kobbelt et al. [8] yields the best results. It minimizes the thin plate energy of the mesh and produces smooth surfaces while causing less volume shrinkage in thin areas compared to other algorithms.
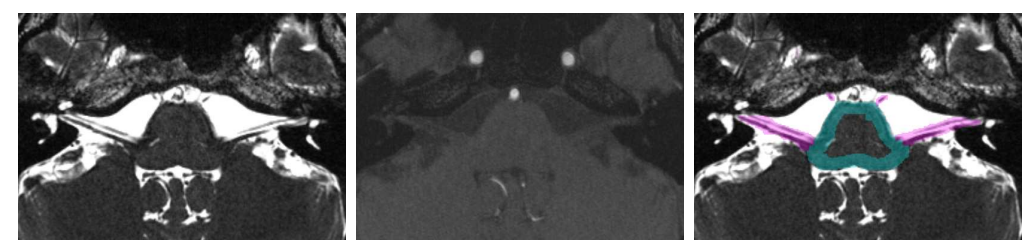

Fig. 1. Corresponding CISS (left), TOF (middle) and segmented CISS (right) slices 
Fig. 2. Error-bounded smoothing. From left to right: original polyline; close-up of the constrained smoothing step; smoothed polyline.
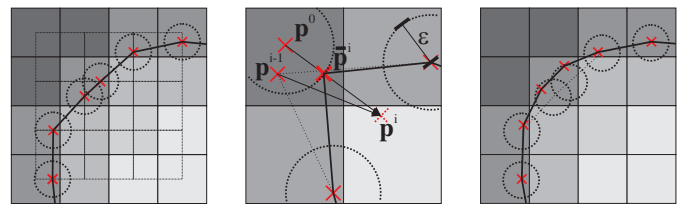

In order to bound the error introduced by mesh smoothing, we constrain vertex movement to a small sphere (we use a diameter of $\epsilon=0.3 \mathrm{~mm}$ in our implementation) around the initial vertex positions $\mathbf{p}_{j}^{0}$ as depicted in figure 2 : During each smoothing iteration, we compute the position $\mathbf{p}_{j}^{i}$ satisfying the second order umbrella equation for each vertex $\mathbf{p}_{j}$. If $\mathbf{p}_{j}^{i}$ lies outside the $\epsilon$-ball of $\mathbf{p}_{j}^{0}$, the vertex is projected back onto the sphere along the line from $\mathbf{p}_{j}^{0}$ to $\mathbf{p}_{j}^{i}$.

\subsection{Distance field computation}

To enable distance computations between nerves and vessels, we compute a distance field for the vascular structures. For this purpose, we create a discrete distance volume data set $\mathcal{D}$ the size of the MR-TOF volume and initialize all voxels $v$ with distance $\mathcal{D}(v)=\infty$. Then, we compute the voxels of $\mathcal{D}$ that are intersected by the arteries mesh and assign them the distance zero (Fig. 3a) and put all zero-distance voxels into a queue (active front). For each voxel in active front we store the closest zero-distance voxel.

In the next step, we propagate the distance information of each voxel $v$ in active front to its 27 direct neighbors $n_{i}$ : If the current distance value $\mathcal{D}\left(n_{i}\right)$ of a neighbor voxel $n_{i}$ is larger than the distance $d$ between $n_{i}$ and the nearest zero-distance voxel of $v, \mathcal{D}\left(n_{i}\right)$ is set to $d$ and $n_{i}$ is added into the active front. After all neighbors of $v$ have been processed, $v$ is removed from the active front queue. This step is iterated until the queue active front is empty (Fig. 3).
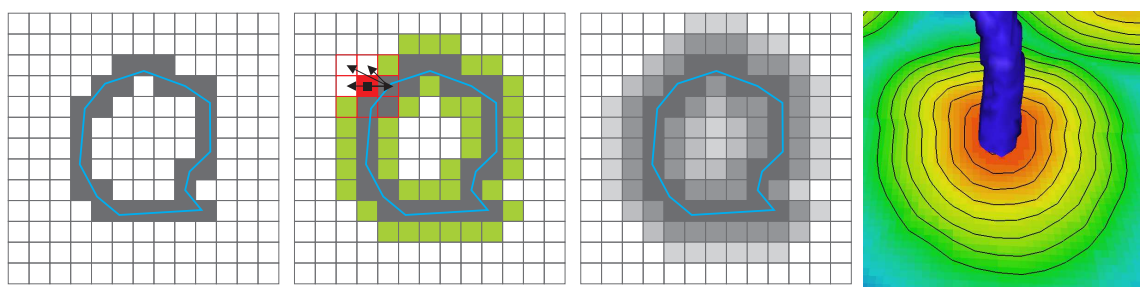

Fig. 3. Distance field computation. From left to right: zero-distance voxels; active front (green) and distance propagation; final distance volume; slice of a 3-D example. 


\subsection{Visualization}

We render the previously extracted polygonal models using standard mesh rendering techniques. The visualization of potential nerve-vessel contacts is further improved by color-coding the mesh of the nerves (Figs. 4, 5): for each vertex of the nerves' mesh, we look up the distance to the vessels' mesh using the distance field $\mathcal{D}$. The vertices are then colored according to the respective distances. Values smaller than $1 \mathrm{~mm}$ are rendered in dark gray, distances between 1 and $3 \mathrm{~mm}$ are colored red to yellow and distances larger than $3 \mathrm{~mm}$ are colored yellow.

\section{Results}

The presented methods were so far used for pre-operative planning in 6 cases $(4 \times \mathrm{TN}, 2 \times \mathrm{HS})$. Data processing and analysis took roughly 2 hours: MR imaging (25 min), segmentation (1 hour), registration (10 min), mesh extraction, smoothing and distance field computation $(15 \mathrm{~min})$. The proposed polygonal visualization ensures interactive rendering even on workstations without highend graphic cards. Renderings are shown for two of the examined cases in figure 4 and figure 5. In all cases, the identified contact locations were verified during surgery.

\section{Discussion}

The presented technique improves diagnosis of nerve-vessel contacts and leads to a more precise localization and quantitative analysis in pre-operative planning. Compared to traditional direct volume visualization (e.g. 3D-texture-slicing) (Fig. 4(left)), the perception of distances and spatial relationships is significantly improved by rendering color-coded polygonal models of the related structures.

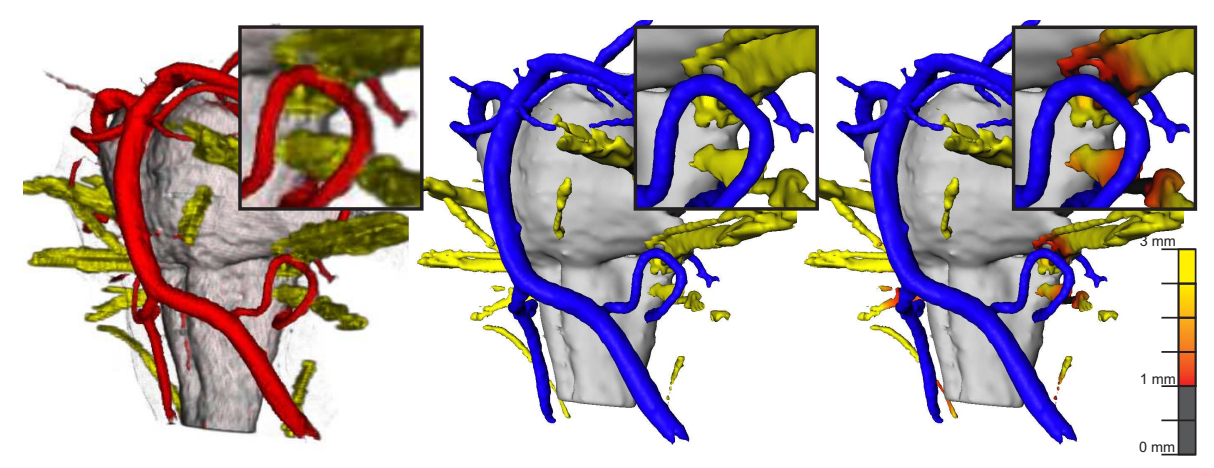

Fig. 4. Comparing rendering quality: conventional volume rendering of fused CISSTOF volume (left), rendering polygonal models only (middle) and polygonal models with color-coded distance information (right). 
Fig. 5. Case of hemifacial spasm: intraoperative photo (left) and rendering in the same viewing direction showing the offending artery (1) and the related nerve (2) (right).

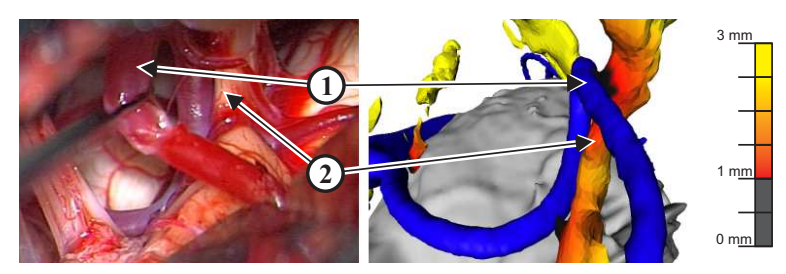

Our approach provides an excellent overview of the target area and allows easy interaction with the delineated structures. Furthermore, it offers the opportunity of non-invasive exploration and classification of the critical nerve-vessel contacts causing NVC syndromes.

During surgery, unnecessary manipulations with the risk of collateral damages leading to neurological deficits can be considerably reduced because of a more straight forward approach to the lesion. Furthermore, this technology can be very useful in large scale epidemiological studies on NVC syndromes. In consequence, the presented technique can support a further classification of neurovascular contacts. Other application fields include the improved information of the patient before the intervention as well as the use in education.

\section{References}

1. Jannetta P. Neurovascular compression in cranial nerve and systemic disease. Ann Surg. 1980;192:518-525.

2. Hastreiter P, Naraghi R, Tomandl B, et al. 3D-visualization and registration for neurovascular compression syndrome analysis. Procs MICCAI. 2002; p. I,396-403.

3. Naraghi R, Hastreiter P, Tomandl B, et al. Three dimensional visualization of neurovascular relationship in the posterior fossa: Technique and clinical application. J Neurosurg. 2004;100(6):1025-35.

4. Akimoto H, Nagaoka T, Nariai T, et al. Preoperative evaluation of neurovascular compression in patients with trigeminal neuralgia by use of three-dimensional reconstruction from two types of high-resolution magnetic resonance. Neurosurgery. 2002;51(4):956-61.

5. Hastreiter P, Vega-Higuera F, Tomandl B, et al. Advanced and standardized evaluation of neurovascular compression syndromes. Procs SPIE. 2004;5:267-274.

6. Vega-Higuera F, Hastreiter P, Fahlbusch R, et al. High performance volume splatting for visualization of neurovascular data. Procs IEEE Vis. 2005; p. 271-278.

7. Miller J, Acar F, Hamilton B, et al. Preoperative visualization of neurovascular anatomy in trigeminal neuralgia. J Neurosurg. 2008;108(3):477-82.

8. Kobbelt L, Campagna S, Vorsatz J, et al. Interactive multi-resolution modeling on arbitrary meshes. Procs SIGGRAPH. 1998; p. 105-114. 